\title{
RESEARCH ON THE PICTURE COLLECTED BY MICROMINIATURE AIRCRAFT TO CONSTRUCT DIGITAL ELEVATION MODEL
}

\author{
Likang Shao ${ }^{1}$, Guangxing Wei ${ }^{2}$, Qiyu Yang ${ }^{3}$, Xisheng $\mathrm{Wu}^{2}$ \\ ${ }^{I}$ Machinery Staff Room Artillery Academy ,HeFei .China Email: \\ mailto:yang_baojiang@163.com. ${ }^{2}$ School of information technology, Southern Yangtze \\ university,Wuxi, China; Email: weigx407@126.com 3. Precision Machinery Department, \\ Shanghai University, Shanghai China,Email:.shaolikang@163.com
}

\begin{abstract}
It is the primary step of the "digital city" that construct tridimensional digital map[1]. We consider that construct it by computer vision base on the characteristic of the image microminiature aircraft collected[2]. But the quantity of the pairs of the characteristic point cannot meet the request to construct Digital Elevation Model. This paper provide a latest correlate matching method base on Epipolar Constrain after introduce the characteristic point extract and matching. the paper adopt a precise Fundamental Matrix estimate algorithm to calculated epipolar equation and increase the matching point's quantity effectively. It has met the real time 's request by experimental verification.
\end{abstract}

Key words: Epipolar Constrain Computer Vision Digital, Elevation Model Fundamental Matrix.

\section{FOREWORD}

Satellite remote sensing and navigate photography adapt to the data source of big region cartography. But the costs of conversion is too large, the cycle of the data acquisition is too long. It is feasible and practicable that that method of construct tridemensional map which use the pictures from the low-level microminiature aerocraft contrapose the current technology and cost. It suitable for the request of construct partly clearly tridimensional digital map because of its spending, flight level and mobility.

This project is supported by "863" project under the grant No.2001AA422160 and No. 2004AA420110

Please use the following format when citing this chapter:

Shao, Likang, Wei, Guangxing, Yang, Qiyu, Wu, Xisheng, 2006, in International Federation for Information Processing (IFIP), Volume 207, Knowledge Enterprise: Intelligent Strategies In Product Design, Manufacturing, and Management, eds. K. Wang, Kovacs G., Wozny M., Fang M., (Boston: Springer), pp. 984-989. 
This research based on the nonstandard aerophotogrammetry picture construct the Digital Elevation Model (DEM). It needs enough right pairs of matching characteristic point to calculate the position's space coordinate. It can calculate the space coordinate by the pairs of matching point's image coordinate and camera's inside and outside parameter. And it also can construct DEM by those space coordinates.

\section{CHACTERISTIC POINT EXTRACT AND MATCHING AND THEIR EXPERIMENT}

\subsection{Characteristic Point Extract}

Make the corner of the image be the character[3]. Harris arithmetic operators differential coefficient's formula:

$$
f_{x}(x, y)=\frac{\partial f}{\partial x}=f(x+1, y)-f(x-1, y) \quad f_{y}(x, y)=\frac{\partial f}{\partial y}=f(x, y+1)-f(x, y-1)
$$

and it introduce the gauss smoothness strain in Harris operator. So in fact the characteristic matrix $\tilde{Q}$ :

$$
\tilde{\boldsymbol{Q}}=\left(\begin{array}{cc}
\sum \tilde{\mathrm{f}}_{\mathrm{x}}^{2} & \sum \tilde{f}_{\mathrm{x}} \mathrm{f}_{\mathrm{y}} \\
\sum \mathrm{f}_{\mathrm{x}} \mathrm{f}_{\mathrm{y}} & \sum \tilde{\mathrm{f}}_{\mathrm{y}}^{2}
\end{array}\right)
$$

Replace the $\tilde{Q}$ for $\mathrm{M}$, the last characteristic matrix $\tilde{Q}$ can be:

$$
M=\left(\begin{array}{ll}
A & C \\
C & B
\end{array}\right)
$$

Harris define the estimate strength factor of the corner:

$$
H(x, y)=\operatorname{det}(M)-k^{*} \operatorname{trace}(M)^{2}
$$


Ahead of schedule it must define the strength criterion $H_{t h r}$. It confirm it be the corner when $H(x, y)>H_{t i r}$, So it can select a comparatively lower $H_{t t r}$ to get enough corner and sort it by the $H(x, y)$ and select definite quantity.

\subsection{Characteristic point matching}

Find out the characteristic point which the same scene in different image project named characteristic point matching. There are many matching method adapt to different condition[4]. The matching means base on the regiondepent make a presumption that the corresponding pixel has similitude brightness. We must consider several neighbor pixel's brightness. for example, make a hypothesis that $\boldsymbol{u}_{i}=(u, v)^{\mathrm{T}}$ be a point of the first image, and its grey scale value be $h_{1}(u, v), u_{j}^{\prime}=\left(u^{\prime}, v^{\prime}\right)^{\mathrm{T}}$ should be any point of the second image, and its grey scale value should be $h_{2}\left(u^{\prime}, v^{\prime}\right)$.Then define that the gradation similarity of the point $\boldsymbol{u}_{i}$ and $\boldsymbol{u}_{j}^{\prime}$ as the gradation correlation value of the neighborhood $\Omega$ :

$$
\sigma_{i j}=\frac{1}{N} \sum_{k, \Omega \Omega}\left(h_{1}(u-k, v-l)-\bar{h}_{1}\right)\left(h_{2}\left(u^{\prime}-k, v^{\prime}-l\right)-\bar{h}_{2}\right)
$$

If the characteristic point $u_{i}$ in first image match to the characteristic point $\boldsymbol{u}_{m}^{\prime}$ in the second image , the $\boldsymbol{u}_{m}^{\prime}$ must be close to the position of $\boldsymbol{u}_{i}$ in the second image because that the camera's displacement and rolling angle will not be very large. If the distance of the two point larger than the radius which define first then make the corresponding correlation coefficient be 0 . It can be consider as a possible matching if the distance of the two point less than this radius and its correlation coefficient less than the defined threshold. it is the mostfrequentlyused means that the regional correlation coefficient(LACC) to determine whether the two point matching in practice : $c_{i j}=\frac{\sigma_{i j}}{\sqrt{\sigma_{1}^{2} \sigma_{2}^{2}}}$.

It is necessary to presumption that it is similar that the characteristic excursion vector in a small neighborhood for the sake of improving the matching effect. So it used weighting function to calculate the matching INTENSIVE of the point multitude which got from the region gradation correlation method. We can presume that there is a pair of matching point 
$\boldsymbol{u}_{i}$ and $\boldsymbol{u}_{i}^{\prime}$. If there is a point $\boldsymbol{u}_{k}$ nearby $\boldsymbol{u}_{i}$ in the first image then the relation between the point $\boldsymbol{u}_{i}^{\prime}$ match to $\boldsymbol{u}_{k}$ in the second image similar to the relation between $\boldsymbol{u}_{i}$ and $\boldsymbol{u}_{k}$. It can express as:

$$
\mu_{(i j, k l)}=\frac{\left(\boldsymbol{u}_{k}-\boldsymbol{u}_{i}\right) \bullet\left(\boldsymbol{u}_{l}^{\prime}-\boldsymbol{u}_{j}^{\prime}\right)}{\max \left(\left\|\boldsymbol{u}_{k}-\boldsymbol{u}_{i}\right\|,\left\|\boldsymbol{u}_{l}^{\prime}-\boldsymbol{u}_{j}^{\prime}\right\|\right)^{2}}
$$

We call it as discommender when $\mu_{t(t k j)}<0 . p_{n}$ express the percent of those discommenders in those matching candidate .It define matching intensity as:

$$
s_{i j}=c_{i j}\left(\sum_{k \in \Omega} \max _{l \in \Omega}\left(c_{k l} \mu_{(i j, k l)} f_{i k}\right)+\sum_{l \in \Omega} \max _{k \in \Omega}\left(c_{k l} \mu_{(i j, k l)} f_{j l}\right)\right)\left(1-p_{n}\right)
$$

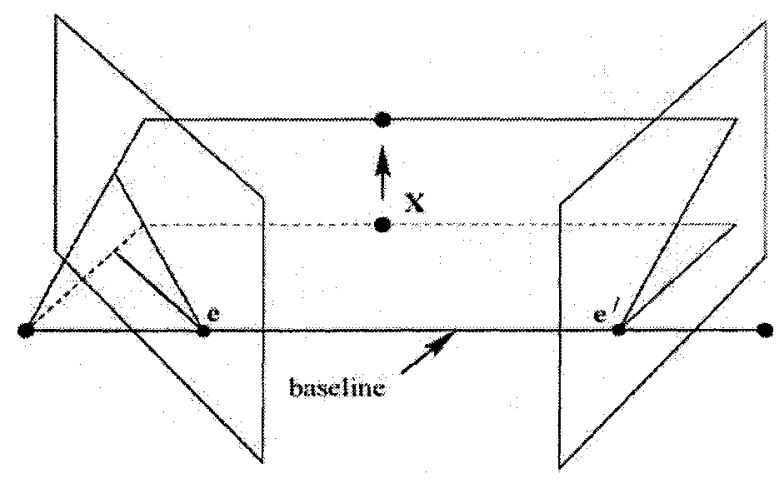

Figure 1. the geometry relation of the two picture

\section{FUNDAMENTAL MATRIX CALCULATE AND ITS EXPERIMENT}

AsFigure 1 noted, it take two picture from two view point. The plane which overpast the two viewpoint intersect with the two picture can get two line (base line). Different base line intersect with each other on one point(base point) in one picture. The corresponding point of Any image point must in it's base line of other picture. It can be recount by Fundamental matrix in algebra. The Fundamental matrix is a three row three rang matrix, 
and it is homogeneous and Its rank is two so it has seven free degree. Any pair of corresponding points $X, X^{\prime}$ and Fundamental Matrix satisfied:

$$
\mathbf{x}^{\prime T} \mathbf{F x}=0
$$

Given the Fundamental Matrix, the corresponding base line of the image point can be ascertain as:

$$
\mathbf{l}^{\prime}=\mathbf{F x}, \quad \mathbf{l}=\mathbf{F}^{T} \mathbf{x}^{\prime}
$$

The Fundamental Matrix can be estimate from the corresponding point. For the given points $(x, y)$ and $\left(x^{\prime}, y^{\prime}\right)$, it establish linear equation as:

$$
\left[\begin{array}{lllllllll}
x^{\prime} x & x^{\prime} y & x^{\prime} & y^{\prime} x & y^{\prime} y & y^{\prime} & x & y & 1
\end{array}\right] \mathbf{f}=0
$$

So it can get ascertain 8 independence parameter of the Fundamental Matrix from 8 pairs of corresponding point. If given the pair of corresponding point $\mathbf{X}$, and the Fundamental Matrix, it can get the he pair of corresponding point $\mathbf{x}^{\prime}$.

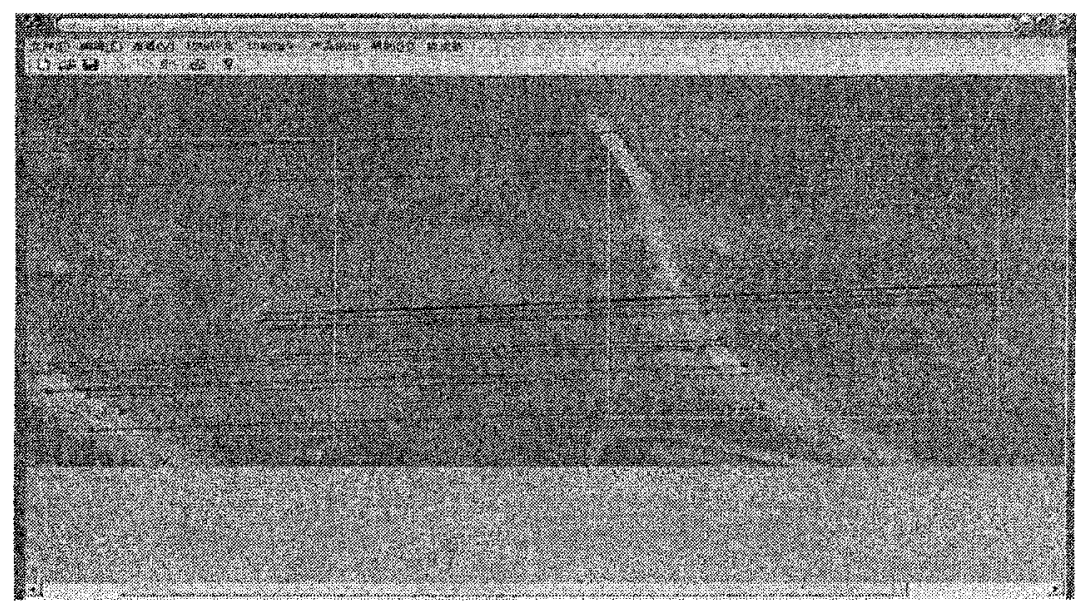

Figure 2. Increase more character base on Epipolar

\section{CONSTRUCT DEM AND DISPLAY}

It should write the elevation correspond to the net-point to a file follow the format which VirtuoZo System submitted, named DEM(Digital Elevation Model) (See Figure 3). 


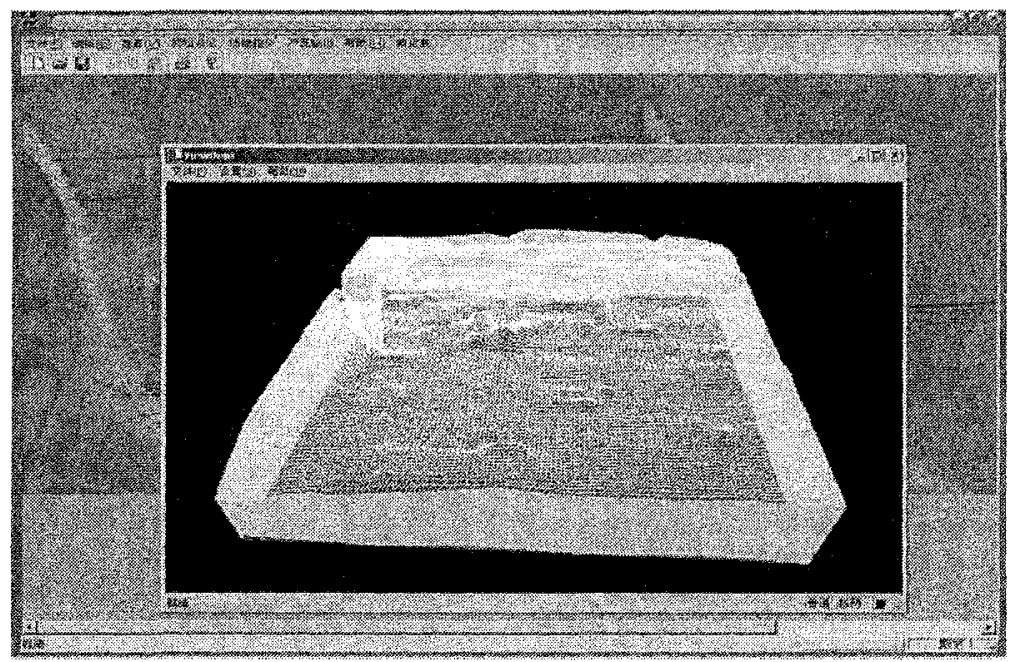

Figure 3. display Dem

\section{SUMMARIZE}

It is the primary step of the "digital city" that construct tridimensional digital map, We complete the step of increase characteristic point by computer vision because of the specialty of the image collected by microminiature aircraft. It also disposed reference point in fieldwork and counted relevant parameter in office work. It did a favorable support to the next work of construct DEM. The experiment proved the effectiveness and feasibility of this algorithm. And the speed of algorithm's perform meet the request of real time.

\section{REFERENCES}

1. Liu, Lin Huang, Ying, (2004.12), How to make the development strategies for digital city, Journal of Harbin Institute of Technology (New Series), vol. 11, pp. 649-653

2. Besl, Paul J. (1998.4.21), Geometric Modeling and Computer Vision, Research Publication - General Motors Research Laboratories, n 6248, Apr 21, 1988,

3. Dong, Mingli Zhou, Xiaogang; Zhu, Lianqing; Lu, Naiguang; Sun, Yunan,(2005,12), Automated image matching with coded points in stereovision measurement, Chinese Journal of Mechanical Engineering (English Edition), vol:18, n 3 ,pp: 453-456

4. Zhong, Hui-Xiang Feng, Yue-Ping; Pang, Yun-Jie. "Robust estimation of the fundamental matrix based on an error model" 2005 International Conference on Machine Learning and Cybernetics, ICMLC 2005, 2005, p 5082-5087 